\title{
Scoliosis corrective force estimation from the implanted rod deformation using 3D-FEM analysis
}

\author{
Yuichiro Abe ${ }^{1 *}$, Manabu Ito ${ }^{2}$, Kuniyoshi Abumi ${ }^{3}$, Hideki Sudo ${ }^{4}$, Remel Salmingo ${ }^{5}$, Shigeru Tadano ${ }^{5}$ \\ From 10th International Research Society of Spinal Deformities (IRSSD) \\ Sapporo, Japan. 29 June - 2 July 2014
}

\begin{abstract}
Background: Improvement of material property in spinal instrumentation has brought better deformity correction in scoliosis surgery in recent years. The increase of mechanical strength in instruments directly means the increase of force, which acts on bone-implant interface during scoliosis surgery. However, the actual correction force during the correction maneuver and safety margin of pull out force on each screw were not well known. In the present study, estimated corrective forces and pull out forces were analyzed using a novel method based on Finite Element Analysis (FEA).

Methods: Twenty adolescent idiopathic scoliosis patients (1 boy and 19 girls) who underwent reconstructive scoliosis surgery between June 2009 and Jun 2011 were included in this study. Scoliosis correction was performed with $6 \mathrm{~mm}$ diameter titanium rod (Ti6Al7Nb) using the simultaneous double rod rotation technique (SDRRT) in all cases. The pre-maneuver and post-maneuver rod geometry was collected from intraoperative tracing and postoperative 3D-CT images, and 3D-FEA was performed with ANSYS. Cobb angle of major curve, correction rate and thoracic kyphosis were measured on X-ray images.
\end{abstract}

Results: Average age at surgery was 14.8, and average fusion length was 8.9 segments. Major curve was corrected from 63.1 to 18.1 degrees in average and correction rate was $71.4 \%$. Rod geometry showed significant change on the concave side. Curvature of the rod on concave and convex sides decreased from 33.6 to 17.8 degrees, and from 25.9 to 23.8 degrees, respectively. Estimated pull out forces at apical vertebrae were $160.0 \mathrm{~N}$ in the concave side screw and $35.6 \mathrm{~N}$ in the convex side screw. Estimated push in force at LIV and UIV were $305.1 \mathrm{~N}$ in the concave side screw and $86.4 \mathrm{~N}$ in the convex side screw.

Conclusions: Corrective force during scoliosis surgery was demonstrated to be about four times greater in the concave side than in convex side. Averaged pull out and push in force fell below previously reported safety margin. Therefore, the SDRRT maneuver was safe for correcting moderate magnitude curves. To prevent implant breakage or pedicle fracture during the maneuver in a severe curve correction, mobilization of spinal segment by releasing soft tissue or facet joint could be more important than using a stronger correction maneuver with a rigid implant.

\section{Background}

Development of novel correction maneuvers and improvement of material property in spinal instrumentation have resulted in better deformity correction in scoliosis surgery in recent years [1,2]. Rod rotation maneuver and direct vertebral rotation technique using thoracic pedicle screw

\footnotetext{
* Correspondence: menchi@athena.ocn.ne.jp

'Department of Orthopaedic Surgery, Wajokai Eniwa Hospital, Eniwa, Hokkaido, Japan

Full list of author information is available at the end of the article
}

have provided better scoliosis correction, and use of rigid instruments such as cobalt-chromium alloy rod have also accelerated these trends of aggressive correction. The use of these aggressive correction maneuver could induce the increase of force exerted on bone-implant interface during scoliosis surgery. There were many biomechanical studies about holding strength of spinal instruments [3-6]. As regards corrective force exerted on the spinal instruments, several biomechanical models were developed to simulate scoliosis correction in-vitro [7-9]. However, there were 
only few reports which estimated the actual corrective force during correction maneuver in vivo $[10,11]$. We have developed a novel method for estimating corrective force acting on the spine by investigating the geometrical change of implanted rod in scoliosis surgery $[12,13]$. In the present study, estimated corrective forces and pull out forces exerted on the pedicle screw were analyzed using a novel method based on finite element analysis.

\section{Methods}

Twenty adolescent idiopathic scoliosis patients ( 1 boy and 19 girls) who underwent reconstructive scoliosis surgery between June 2009 and June 2011 were included in this study. All 20 patients had Type 1 curve. 12 patients had Type 1A curve, 4 had Type 1B curve and 4 fad Type $1 \mathrm{C}$ curve. Curve magnitude of major thoracic curve was $63.1 \pm 6.8$ degrees in average (range: $53-74$ ). Scoliosis correction was performed with $6 \mathrm{~mm}$ diameter titanium rod (Ti6Al7Nb) using the simultaneous double rod rotation technique (SDRRT) in all cases [14]. The pre-maneuver and post-maneuver rod geometry was collected from intraoperative tracing and postoperative $3 \mathrm{D}-\mathrm{CT}$ images, and the changes in the rod curvature were measured (Fig.1). The initial geometry of implant rod was measured from the actual rod used before surgery. Highresolution photograph was taken before insert the rod, and liner model with $6 \mathrm{~mm}$ diameter was created using CAD software Solidworks 2010 (Dassault Systemes, Massachusetts, USA). The implant rod geometry after surgery was obtained a week after the surgical operation using Aquilion 64 CT scanner (Toshiba Medical Systems Corporation, Tochigi, Japan). The slice thickness was $0.5 \mathrm{~mm}$. The images were imported into Solidworks 2010 to measure the $3 \mathrm{D}$ geometry and deformation. Cobb angle of major curve, correction rate and thoracic kyphosis were measured on X-ray images. The study was approved by the Ethical Committee for Clinical Research at Wajokai Eniwa Hosiptal (approval date: April 1, 2011).

\section{FEM}

3D-CAD model of rod geometry was generated and Finite Element Analysis (FEA) was performed using ANSYS 11.0 according to the previously developed method reported by Salmingo et al [12,13]. Material properties of this rod are Young's Modulus $(E)$, yield stress $(\sigma Y)$, yield strain $(\varepsilon Y)$ and hardening coefficient $(\mathrm{H})$ equal to105 $\mathrm{GPa}, 900 \mathrm{MPa}$, $8.57 \times 10-3$ and $2.41 \mathrm{GPa}$, respectively.

A zero force $F_{i}$ ( $i=$ no. of screws $)$ was applied initially to the corresponding location of each screw on the rod geometry before surgery. An elasto-plastic deformation analysis was performed. The displacement vector $\overrightarrow{\mathrm{e}}_{i}$ required to attain the location of each screw after surgery was used in the iterative process. The applied forces to the rod before surgery were replaced by adding

\section{Concave Convex}

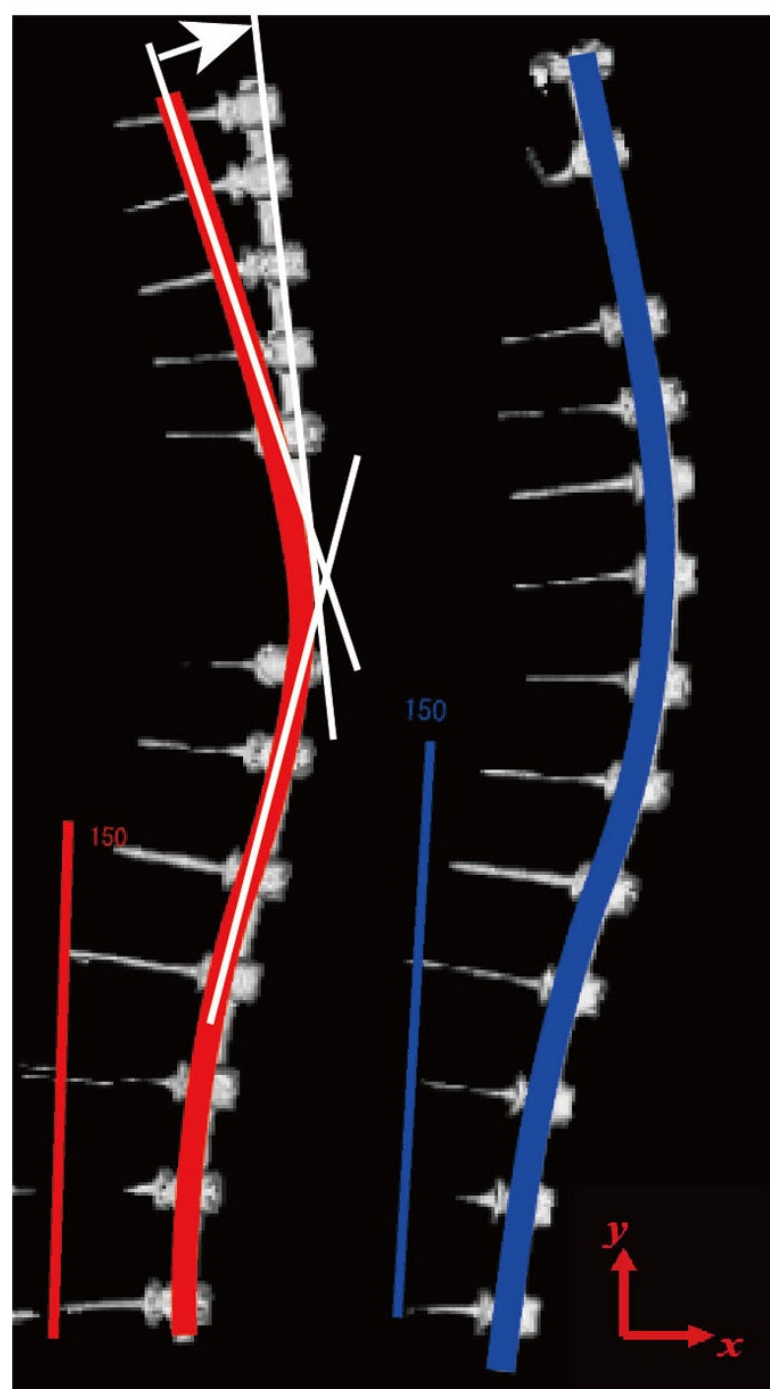

Figure 1 Collection of rod geometry. The rod geometry was collected from intraoperative tracing and postoperative 3D-CT images. Rod contour decreased in postoperative 3D-CT (arrow).

the value of the displacement vector $\overrightarrow{\mathrm{e}}_{i}$. The whole process was iterated until the displacement vector $\overrightarrow{\mathrm{e}}_{i}$ was minimized or the evaluating function was met. The evaluating function is defined as the sum of the squares of displacement vector $\overrightarrow{\mathrm{e}}_{i}$ expressed in Eq. (1). The force iteration process and the finite element deformation analysis was stopped when the evaluating function was less than $\mu$ (where $\mu=0.5$ ). The rod model was deformed the same rod geometry after surgery because the displacement vector $\overrightarrow{\mathrm{e}}_{i}$ was minimized or close to zero. 


$$
\sum_{i=1}^{n}\left|\overrightarrow{\mathrm{e}}_{i}\right|^{2}<\propto
$$

Push out or push in forces exerted on each screw on the bilateral side were estimated using FEA. The calculated forces represent also to the pullout and push-in forces acting at the vertebrae of the scoliosis patient since the implant is directly connected to the vertebra. The magnitudes of pullout and push-in forces were also estimated from the applied forces. The pullout or push-in force was defined as the pulling or pushing force acting normal to the implant curvature at the local coordinate plane $(y-x$ plane since the rod is bent at one plane), Fig. 1. The pullout or push-in force was computed using the reaction force (i.e. opposite force acting at the spine) of the computed corrective force that deformed the rod during scoliosis surgery. The rod geometry was used to define the direction of the pullout or push-in force because its curvature constitutes the spine curvature after the surgical treatment of scoliosis. The rod geometry was approximated by quintic polynomial function using the previous method [13]. A tangent angle $\beta$ that is orthogonal to the pullout or push-in force axis was computed by evaluating the derivative of quintic polynomial function evaluated for location of each screw. The reaction force vector was resolved into component (Reaction force $x \operatorname{Cos} \beta$ ), equal to the magnitude of pullout or push-in force acting at the corresponding fixation level.

\section{Results}

\section{Measurement of radiological parameters}

Average age at surgery was 14.8 and average fusion length was 8.9 segments. Implant density in the current patients were 1.79 (range: 1.6 -2.0). Major curve was corrected from 63.1 to 18.1 degrees in average and correction rate was $71.4 \%$. Rod geometry showed significant change on the concave side. Curvature of the rod on the concave and convex sides decreased from 33.6 to 17.8 degrees, and from 25.9 to 23.8 degrees, respectively (Fig. 2).

\section{FEM}

Estimated pull out forces at apical vertebrae were $160.0 \pm 81.1 \mathrm{~N}$ in the concave side screw and $35.6 \pm$ $15.1 \mathrm{~N}$ in the convex side screw. Estimated push in force at LIV and UIV were $305.1 \pm 131.6 \mathrm{~N}$ in the concave side screw and $86.4 \pm 44.0 \mathrm{~N}$ in the convex side screw. Forces exerted on each screw, estimated using FEA in the representative case are shown in figure 3. We also evaluated the relationship between screw density and forces on screws (Fig. 4). In the presented case, as the number of screws decreased, the pull out force significantly increased. Pull out force at the apical level on the concave side was 2.5 fold greater in the half level inserted model compared to the all level inserted model, and 4.1 fold greater in the apex only model (Fig. 5).

\section{Discussion}

Essentially, corrective force cannot be exerted on the spinal implant beyond the limit of anchor holding strength during scoliosis surgery. If corrective force reaches the limit of anchor strength, implant breakage or bony fracture reported as "screw plowing" could occur [9]. According to the results of the present study, averaged pull out and push in force exerted on each screw during SDRRT fell below previously reported safety margin [6], and therefore this maneuver was demonstrated to be safe for correcting a moderate magnitude curve. To

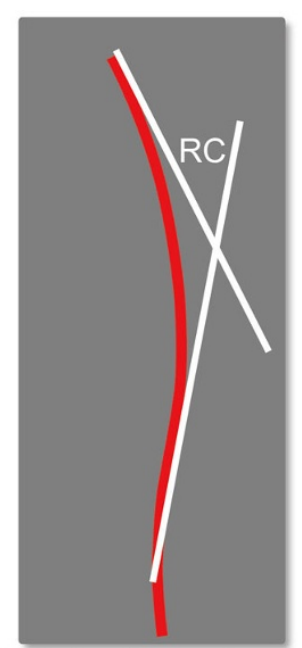

RC: Rod curvature

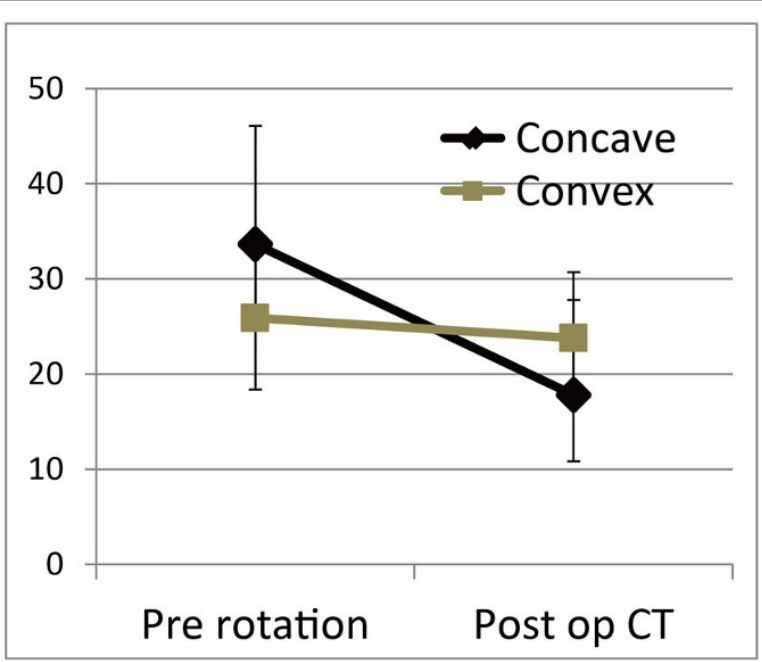

Figure 2 Change in rod geometry. Rod geometry showed significant change on the concave side compared with convex side. 


\section{Concave Convex}
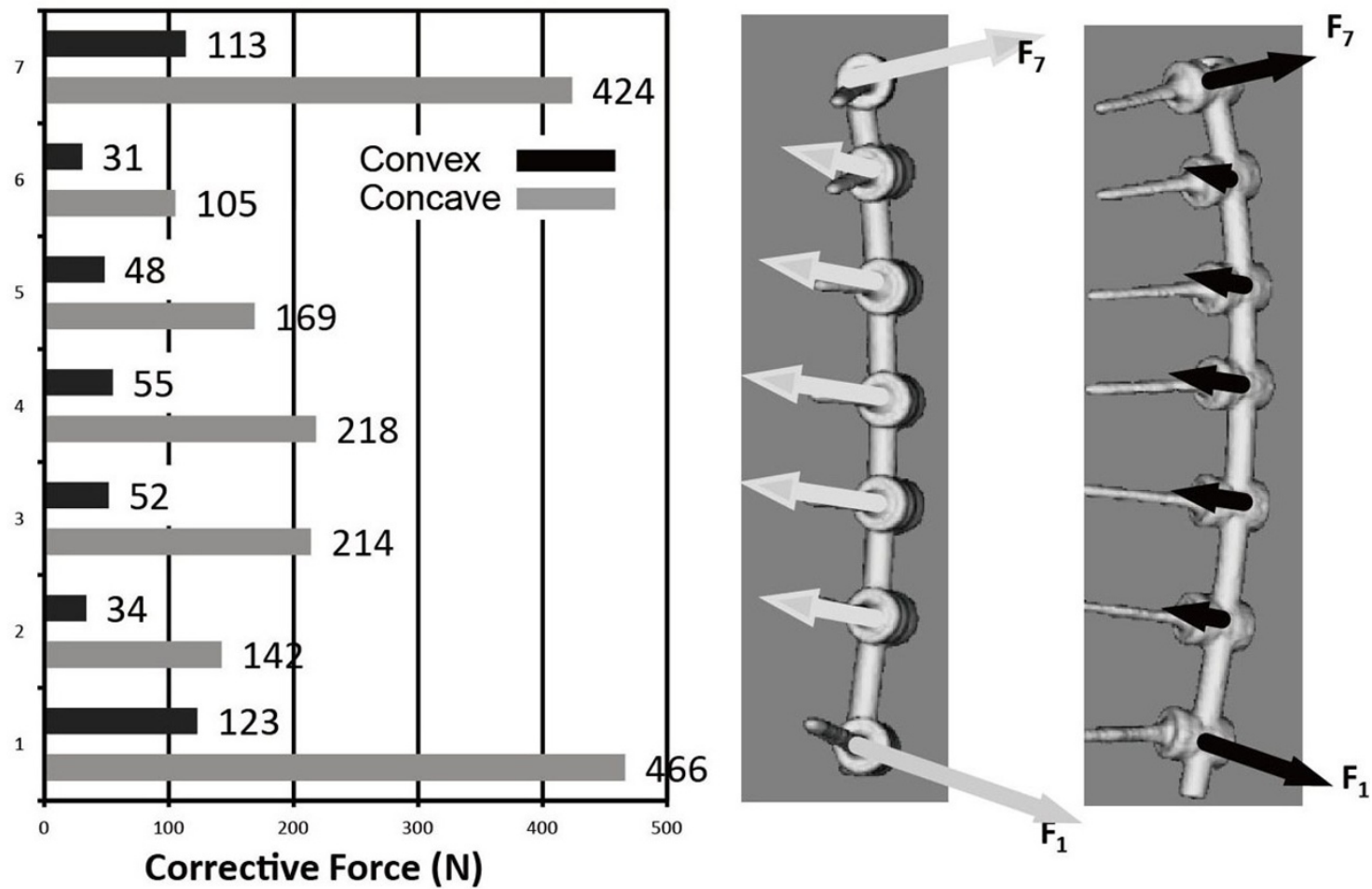

Figure 3 Estimated forces on screws in representative case. Estimated corrective force was four times greater in the concave side than in convex side. Pull out force was greatest in the apical level $(218 \mathrm{~N})$ with smooth transition from one to another and pushing-in forces were high on pedicle screws at the both ends of rod.

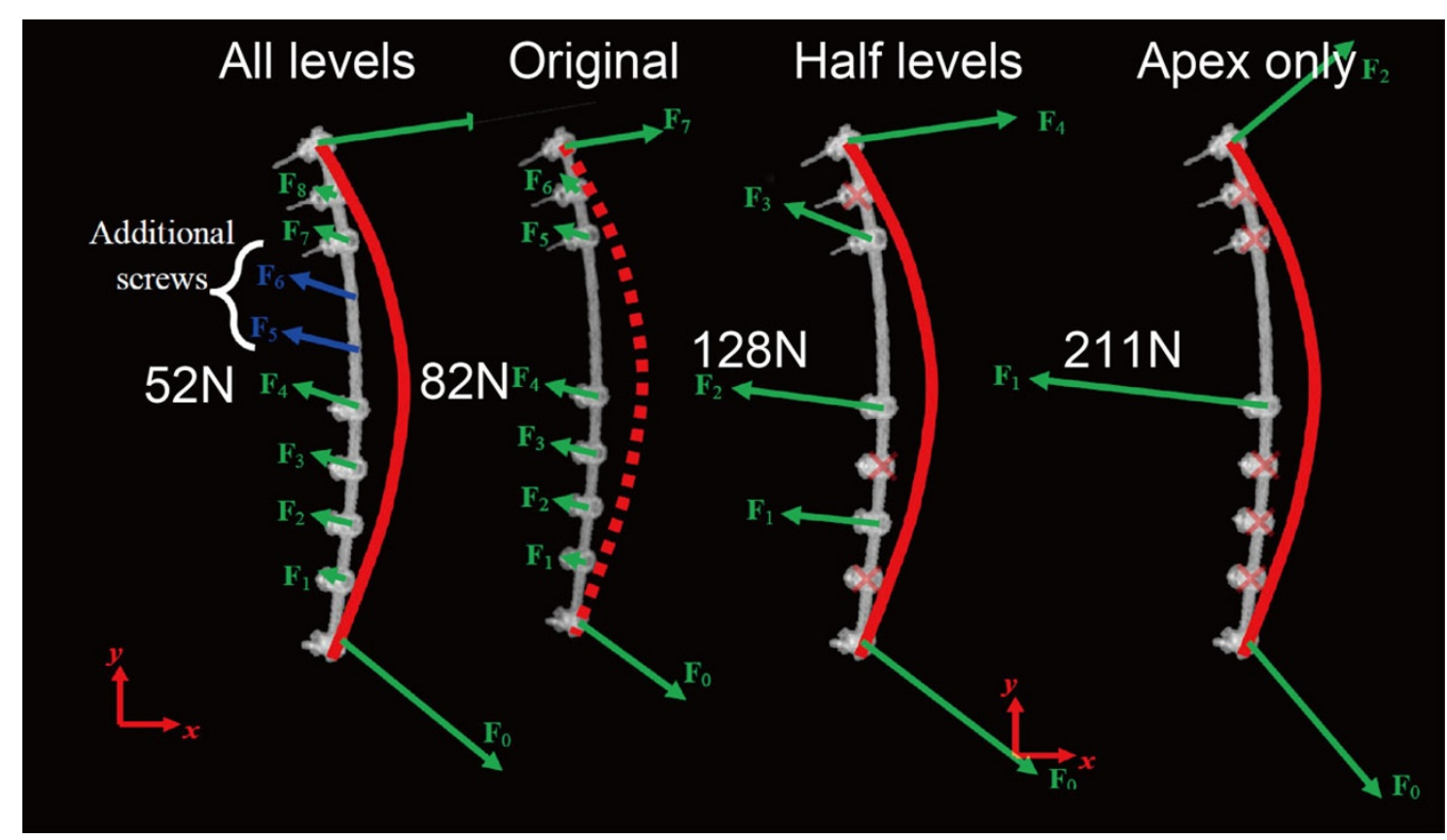

Figure 4 Simulation model of different screw density. As the number of screws decreased, the pull out force exerted on the apical screw significantly increased. 


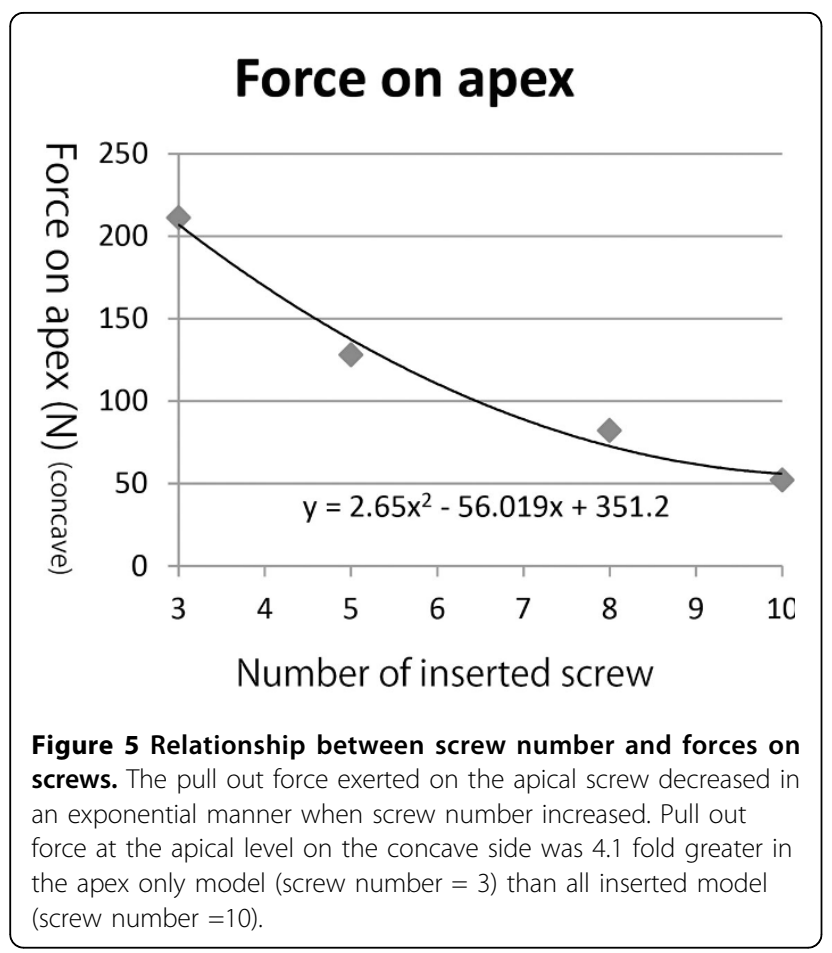

prevent implant breakage or pedicle fracture during maneuver in more severe curve correction, destabilization of spinal segment by releasing soft tissue or facet joint could be more important rather than the use of excessive correction maneuver with rigid implant.

Less screws tend to be used in scoliosis surgery for economical reasons in recent years, and correction rates have been reported as not significantly different between the less density (1.0-1.25) group and the high density (1.752.0) group [15]. Reduction of the implant density, however, also means the load exerted on each anchor is increased. Our simulation study showed that pull out force exerted on the screw in the apical vertebra increased 2.5 fold when screw density decreased from 2.0 to 1.0. To solve these problems, we can use the current simulation technology to estimate in-vivo forces on each spinal implant, to find out the safety margin of implant forces and to determine the best settings of anchors and correction maneuvers for scoliosis correction.

\section{Conclusions}

Corrective force during correction maneuver in scoliosis surgery and pull out forces acting on each pedicle screw were analyzed using a novel method based on Finite Element Analysis. Computational simulation based on in-vivo data makes difficult scoliosis surgery scientifically validated, which can improve safety and effectiveness of deformity correction.

This is the extended abstract of IRSSD 2014 program book [16].

\section{Consent to participate}

Consent to publish for the article's content and images provided as well as consent to participate was obtained from the patients and patient's parents for this study

\section{Competing interests}

The authors declare that they have no competing interests.

\section{Authors' contributions}

Yuichiro Abe has made contributions to conception and design, and has been involved in drafting the manuscript. Remel Salmingo, Hideki Sudo and Kuniyoshi Abumi have made contributions to acquisition and analysis of data. Manabu Ito and Shigeru Tadano have made contribution to study design, revising manuscript and have given final approval of the version to be published.

\section{Declarations}

Publication charges for this collection were funded by IRSSD 2014 Sapporo. This article has been published as part of Scoliosis Volume 10 Supplement 2, 2015: Research into Spinal Deformities 9: Short Papers from the IRSSD 2014 Meeting. The full contents of the supplement are available online at http:// www.scoliosisjournal.com/supplements/10/S2.

\section{Authors' details}

${ }^{1}$ Department of Orthopaedic Surgery, Wajokai Eniwa Hospital, Eniwa, Hokkaido, Japan. ${ }^{2}$ Department of Orthopaedic Surgery, Hokkaido Medical Center, Sapporo, Hokkaido, Japan. ${ }^{3}$ Department of Orthopaedic Surgery, Sapporo Orthopaedic Hospital, Sapporo, Hokkaido, Japan. ${ }^{4}$ Department of Orthopaedic Surgery, Hokkaido University, Sapporo, Hokkaido, Japan. ${ }^{5}$ Division of Human Mechanical Systems and Design, Faculty of Engineering, Hokkaido University, Sapporo, Hokkaido, Japan.

Published: 11 February 2015

\section{References}

1. Lee SM, Suk SI, Chung ER: Direct vertebral rotation: a new technique of threedimensional deformity correction with segmental pedicle screw fixation in adolescent idiopathic scoliosis. Spine (Phila Pa 1976) 2004, 29:343-349.

2. Suk SI, Kim JH, Kim SS, Lim DJ: Pedicle screw instrumentation in adolescent idiopathic scoliosis (AIS). Eur Spine J 2012, 21:13-22.

3. Butler TEJ, Asher MA, Jayaraman G, Nunley PD, Robinson RG: The strength and stiffness of thoracic implant anchors in osteoporotic spines. Spine (Phila Pa 1976) 1994, 19:1956-1962.

4. GaGayet LE, Pries P, Hamcha H, Clarac JP, Texereau J: Biomechanical study and digital modeling of traction resistance in posterior thoracic implants. Spine (Phila Pa 1976) 2002, 27:707-714.

5. Murakami H, Yamazaki K, Attallah-Wasif ES, Tsai KJ, Shimamura T, Hutton WC: A biomechanical study of 3 different types of sublaminar wire used for constructs in the thoracic spine. J Spinal Disord Tech 2006, 19:442-446.

6. Pfeiffer M, Gilbertson LG, Goel VK, Griss P, Keller JC, Ryken TC, et al: Effect of specimen fixation method on pullout tests of pedicle screws. Spine (Phila Pa 1976) 1996, 21:1037-1044.

7. Aubin CE, Labelle H, Chevrefils C, Desroches G, Clin J, Eng AB: Preoperative planning simulator for spinal deformity surgeries. Spine (Phila Pa 1976) 2008, 33:2143-2152.

8. Aubin CE, Petit Y, Stokes IA, Poulin F, Gardner-Morse M, Labelle H: Biomechanical modeling of posterior instrumentation of the scoliotic spine. Comput Methods Biomech Biomed Engin 2003, 6:27-32.

9. Wagner MR, Flores JB, Sanpera I, Herrera-Soto J: Aortic abutment after direct vertebral rotation: plowing of pedicle screws. Spine (Phila Pa 1976) 2011, 36:243-247.

10. Wang $X$, Aubin CE, Crandall D, Labelle H: Biomechanical comparison of force levels in spinal instrumentation using monoaxial versus multi degree of freedom postloading pedicle screws. Spine (Phila Pa 1976) 2011, 36:E95-E104.

11. Wang $X$, Aubin CE, Crandall D, Labelle H: Biomechanical modeling and analysis of a direct incremental segmental translation system for the 
instrumentation of scoliotic deformities. Clin Biomech (Bristol, Avon) 2011, 26:548-555.

12. Salmingo R, Tadano S, Fujisaki K, Abe $Y$, Ito M: Corrective force analysis for scoliosis from implant rod deformation. Clin Biomech (Bristol, Avon) 2012, 27:545-550.

13. Salmingo RA, Tadano S, Fujisaki K, Abe $Y$, Ito M: Relationship of forces acting on implant rods and degree of scoliosis correction. Clin Biomech (Bristol, Avon) 2012.

14. Ito M, Abumi K, Kotani Y, Takahata M, Sudo H, Hojo Y, et al: Simultaneous double-rod rotation technique in posterior instrumentation surgery for correction of adolescent idiopathic scoliosis. J Neurosurg Spine 2010, 12:293-300.

15. Quan GM, Gibson MJ: Correction of main thoracic adolescent idiopathic scoliosis using pedicle screw instrumentation: does higher implant density improve correction? Spine (Phila Pa 1976) 2010, 35:562-567.

16. Yuichiro Abe, et al: Scoliosis corrective force estimation from the implanted rod deformation using 3D-FEM analysis. Scoliosis 2015, 10(Suppl 1):023.

doi:10.1186/1748-7161-10-S2-S2

Cite this article as: Abe et al: Scoliosis corrective force estimation from the implanted rod deformation using 3D-FEM analysis. Scoliosis 2015 10(Suppl 2):S2.

\section{Submit your next manuscript to BioMed Central} and take full advantage of:

- Convenient online submission

- Thorough peer review

- No space constraints or color figure charges

- Immediate publication on acceptance

- Inclusion in PubMed, CAS, Scopus and Google Scholar

- Research which is freely available for redistribution

Submit your manuscript at www.biomedcentral.com/submit 